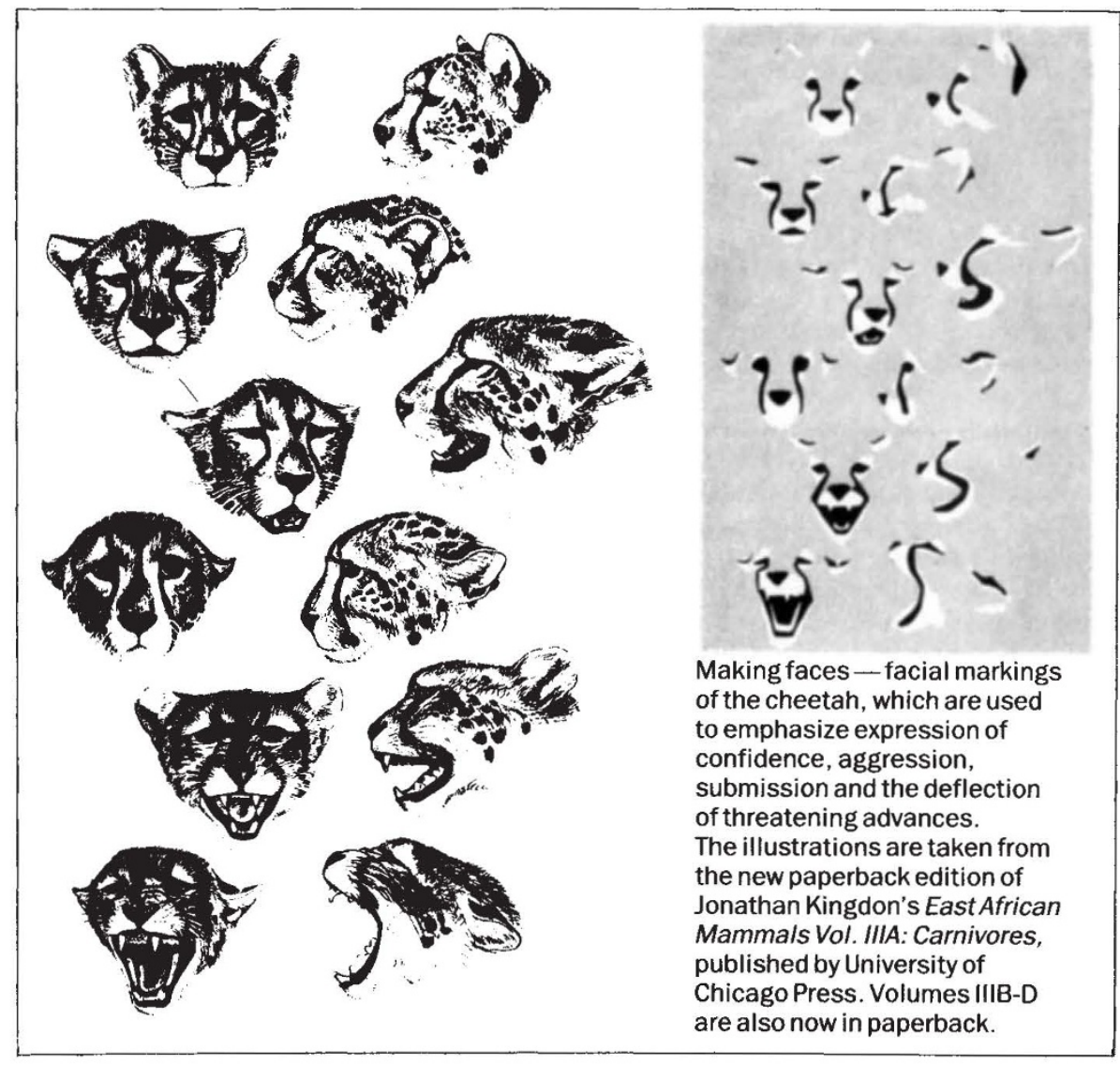

\section{Field work}

\section{Sean Washburn}

Magnetoresistance in Metals. By A. B. Pippard. Cambridge University Press: 1989. Pp.253. £39, \$59.50.

Professor Pippard has expanded on his earlier monograph (Dynamics of Conduction Electrons) in a new book that surveys the broad topic of magnetoresistance in metals. A chapter on fundamental aspects of transport in a magnetic field, pitched at the level of Ziman's Principles of the Theory of Solids, introduces matters, preceding a review of theoretical and experimental work. Classical magnetoresistance is discussed along with illustrative experiments, and then 'semi-classical' effects are surveyed in detail. Untangling of this web of data takes up the bulk of the text, and it is no mean feat; for example even potassium, "the simplest of metals" which has a nearly spherical Fermi surface, exhibits a bewildering variety of magnetoresistance. Finally, Pippard turns to inhomogeneous samples and briefly describes recent results, including electron focusing and resistance fluctuations arising from Aharonov-Bohm effects and coherence of the electrons.

The book proceeds from the simple to the complex; one starts from a familiar base and then is led to attempt interpretation of a great deal of complicated data.
The text is accompanied by diagrams that define notation and illustrate the physics, and by representative data from experiments. Here as in other texts, the depictions of Brillouin zones would have benefited from colour and modern computer graphics. The physics is always deep enough to describe the data and is clearly presented. It is, however, never more complicated than a single particle picture (implicitly from the Fermi liquid) - interaction among the electrons is mentioned only casually in connection with heavyfermion metals.

Does this book meet a need? Yes. In particular the chapter on experiments should be read by anyone attempting to make such measurements, because here Pippard lays down clear warnings about spurious responses and describes methods that avoid them.

I have a few complaints. There is disproportionate citation of work performed in Britain, even after allowing that much of the original research was carried out at the Cavendish Laboratory. There are also quite a few annoying typographical errors (mostly obvious, such as the mislabelling of figures), and occasionally the language and notation are unusual. Nonetheless the book will serve well as background reference for anyone working in this field, and as a concise introduction for anyone entering it.

Sean Washburn is at the IBM Watson Research Center, PO Box 218, Yorktown Heights, New York 10598, USA.

\section{Bringing it all together}

\author{
John Davey
}

Biomembranes: Molecular Structure and Function. By Robert B. Gennis. SpringerVerlag: 1989. Pp.533. \$59, £32.

RoBERT Gennis's Biomembranes is the fourth volume to appear in Springer's Advanced Texts in Chemistry series. The series has an established record of excellence, and this latest addition lives up to expectations. Gennis set out to produce a book that he would like to have had as a student, as well as one that he would find useful as a research scientist. He has achieved both of these goals, bringing together the diverse areas of membrane biology in a manner that is both informative and stimulating.

Here is perhaps the most complete treatment of membrane biology currently available. It covers almost all aspects of the subject, not only structure but also transport, receptors and biogenesis there is something for everyone. Indeed, the author apologizes for the possible inclusion of too much information, and suggests that readers may wish to skip over sections in which they are not interested. The apology is unnecessary. Although I do take Gennis's point, his intention has been to arrange the material to the best effect for teaching purposes. Thus there is a gradual introduction to the structure and composition of membranes, Chapter 1 providing a general survey of the various lipids and proteins, Chapters 2 and 3 dealing with them in greater detail, and Chapters 4 and 5 describing their interaction.

Features of the book I especially liked are the summaries at the end of each chapter - they are true summaries, uncluttered with detail - and the numerous 'boxes' which are used to highlight certain aspects or to cover a particular point in depth without distracting from the flow of the main text. The reference list is a highlight, being comprehensive yet unobtrusive.

My only criticism concerns the figures. Although most of those that do appear are relevant and clear, the book would have benefited from having more of them, and from the inclusion of photographs. Students now expect their textbooks to contain an abundance of high-quality illustrations, and the comparative lack of visual material will, I suspect, lessen the acceptance of Biomembranes for teaching. Given its price, though, this is a book that is likely to be bought largely by libraries and laboratories. It is also one that will be in great demand.

John Davey is in the School of Biochemistry, University of Birmingham, PO Box 363, Birmingham B15 2T, UK. 